Copyright 2001 IEEE. Published in the Proceedings of SIcon'01, November, 2001 in Rosemount, Illinois, USA. Personal use of this material is permitted. However, permission to reprint/republish this material for advertising or promotional purposes or for creating new collective works for resale or redistribution to servers or lists, or to reuse any copyrighted component of this work in other works, must be obtained from the IEEE. Contact: Manager, Copyrights and Permissions / IEEE Service Center / 445 Hoes Lane / P.O. Box 1331 / Piscataway, NJ 08855-1331, USA. Telephone: + Intl. $732-562-3966$.

\title{
The Effects of Heat Treatment on the Magnetic Behavior of Ring-Type Magnetoelastic Torque Sensors
}

\author{
Mark S. Boley, David K. Rigsbee, and Doug A. Franklin \\ Department of Physics, Western Illinois University, Macomb, IL 61455
}

\begin{abstract}
Experiments have been performed to determine the effects of appropriate sequential heat treatment steps on thin maraging steel rings shrink-fitted onto stainless steel shafts for use as magnetoelastic torque transducers. The transducer response was found to be linear and reproducible. Subsequent to heat treatment, the transducer function was remarkably improved, exhibiting a more than twofold increase in sensitivity. By measurements of the axial hysteresis, this enhanced sensitivity can be traced to a corresponding decrease in axial coercive force. Measurement of the circumferential hysteresis also showed a more than fourfold increase in circumferential coercive force, which contributes to improved sensor integrity.
\end{abstract}

\section{Introduction}

Over the past few years, electronic torque sensors utilizing magnetoelastic materials to generate field signals linearly proportional to applied torque (i.e. the name "magnetoelastic torque sensors") have been successfully demonstrated in a variety of applications. One of their primary engineering applications has been in the electronic control of power steering systems and automatic transmissions for the automotive industry [1,2]. In older power steering and automatic transmission systems, most automated functions were hydraulically actuated and mechanically controlled. In either case, the utilization of mechanical sensing devices resulted in the loss of fuel economy and reduced power as functions were often performed at less than optimal times. Recent years have seen the increase of computer controlled functions within the automotive system, with a series of electronic sensors monitoring many aspects of the vehicle's performance with improved economy and power always in view. When the input or output torque can be monitored electronically via transducer signals that lend themselves to direct computer analysis, this results in more precise control of such automotive systems. Unfortunately, the environment in which such electronic sensors must function, if in actual contact to the torqued shafts, would often be quite hostile to their components due to high temperature conditions and the presence of lubricating fluids. However, the magnetoelastic torque transducer can solve these problems, since no physical contact between the sensor itself and the torqued shaft need be maintained. Rather, small magnetic field sensors can be remotely located to collect the field signals generated by the magnetically conditioned portion of the torqued shaft, which itself serves as the magnetoelastic transducer [2,3]. All electronic components can then be safely encapsulated in a small package away from the hostile environment. Similarly, there are a variety of other industrial applications that could benefit from this type of magnetoelastic torque sensing.

Central to the performance of these torque transducers is the uniqueness and stability of the circumferential alignment of the magnetic domains in the sensory ring which is shrinkfitted onto the torqued shaft. This stability is partially provided by the associated tensile "hoop" stress in the sensory ring [4], but a large circumferential coercive force in the material of the ring is also essential to the successful performance and durability of the transducer, as has been previously shown in the literature [5]. While the coercive force is a single-valued function of the chemical, metallurgical, structural, and geometric properties of a sample [6], we herein describe a method of enhancing the circumferential coercive force through a series of heat treatments in one of the major alloys used for the sensory ring. This is found to contribute not only to improved transducer stability, but also to enhanced sensitivity of the sensory ring to the applied torque on the shaft.

\section{Experiment}

The experiments reported here have been performed to determine the effects of appropriate sequential heat treatment steps on two identical thin maraging steel rings (consisting of ferromagnetic composite high-speed steel alloy type C250-containing $18 \%$ nickel by weight, several other trace elements, and remaining balance Fe--with $21.1 \mathrm{~mm}$ outside diameter and $22.1 \mathrm{~mm}$ length with $1.8-\mathrm{mm}$-thick walls) that were shrink-fitted for use as magnetoelastic torque sensors onto non-ferromagnetic stainless steel (type C304-SS) shafts. The stress-induced uniaxial anisotropy ("hoop stress") in the shrink-fitted rings combined with the crystalline anisotropy intrinsic to the ring material, along with its inherently strong circumferential coercive force, serve to stabilize the 
magnetization in opposing circumferential directions in the two halves of the C250 ring, which appropriately conditions the ring for its transducer function. When the ends of the shaft are subjected to an applied torque and a corresponding torsional shearing stress is induced throughout the length of the shafts as well as in the shrink-fitted ring, the magnetic axes of the ring are tilted from a circumferential into a helical orientation, which generates an axial field signal linearly proportional to the applied torque that can be sensed externally to the shaft and the ring. The axial field signals generated from the two halves of the C250 transducer ring will be in opposing directions but of nearly identical magnitude, which allows for two field sensors operating in a differential comparison mode to detect and discard any stray field signals.

In order to enhance the torque transducer function, that is, the field signal sensitivity to applied torque, the C250 transducer rings were subjected to two appropriate sequential heat treatment steps in a helium atmosphere at $843^{\circ} \mathrm{C}$ and $482^{\circ} \mathrm{C}$, for 2 and 3 hours, respectively. In each case, the rings were removed from the stainless steel shafts and placed on a stainless steel mandrel to preserve their shape during the heat treatment and furnace cooling. We obtained a direct CRO screen display of the hysteresis $(\mathrm{B}-\mathrm{H})$ loops of the rings prior to and subsequent to each heat treatment step, while they were removed from the shafts, in order to determine the changes in their axial and circumferential magnetic hysteresis properties. These hysteresis measurements were taken by winding primary and secondary coils about the rings in both the axial and circumferential directions and applying $60 \mathrm{~Hz}$ ac at currents up to $16 \mathrm{~A}$. Additionally, the sensory rings were replaced on the shafts after each heat treatment step for the analysis of the effects of that heat treatment step on the torque transducer function. In each case, the torque load-

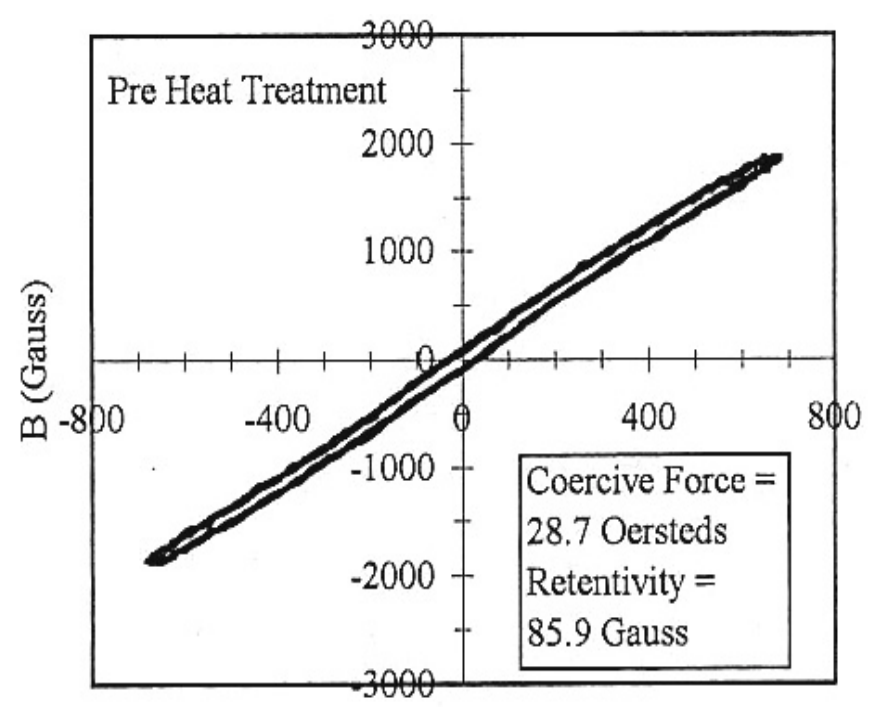

$\mathrm{H}$ (Oersteds)

Fig. 1. A plot of the axial hysteresis loop for the transducer ring prior to heat treatment. ing test was performed to the limits of the linear field signal response to applied torque (shear stress) in either direction. The hysteresis characteristics of a particular ferromagnetic material help determine the utility and/or efficiency of that material for the desired application as a torque sensor. Large retentivity (magnetic induction $\mathrm{B}$-axis intercept) characterizes "hard" iron as used in a permanent magnet, and low coercive force (applied magnetic field $\mathrm{H}$-axis intercept) characterizes "soft" iron as used for a transformer core. For the torque sensor application, a large retentivity and high coercive force are both needed in the circumferential direction to maintain the magnetization of the sensory ring in that direction, while a low retentivity and low coercive force are desirable in the axial direction to allow for maximum torque sensitivity of the transducer ring.

\section{Results and discussion}

In Figures 1 and 2, respectively, are shown the axial hysteresis plots prior and subsequent to the two-step heat treatment procedure for one of the sensory rings (and are typical of both rings). All of the hysteresis loops shown in this paper underwent appropriate background corrections via differential comparison with like data obtained from a paramagnetic aluminum ring. Although our available current did not allow for entire saturation of the axial loops, the effect on the coercive force and retentivity values is minimal as these were observed to undergo only negligible change in the upper regions of applied field. Subsequent to heat treatment, we found a $60 \%$ decrease in the axial coercive force $\left(\mathrm{H}_{\mathrm{k}}\right.$-the $\mathrm{H}$-axis intercept in Oersteds), from 28.7 to $11.3 \mathrm{Oe}$, and an $80 \%$ decrease in the axial retentivity (or remanent magnetization-the B-axis intercept in Gauss), from 85.9 to

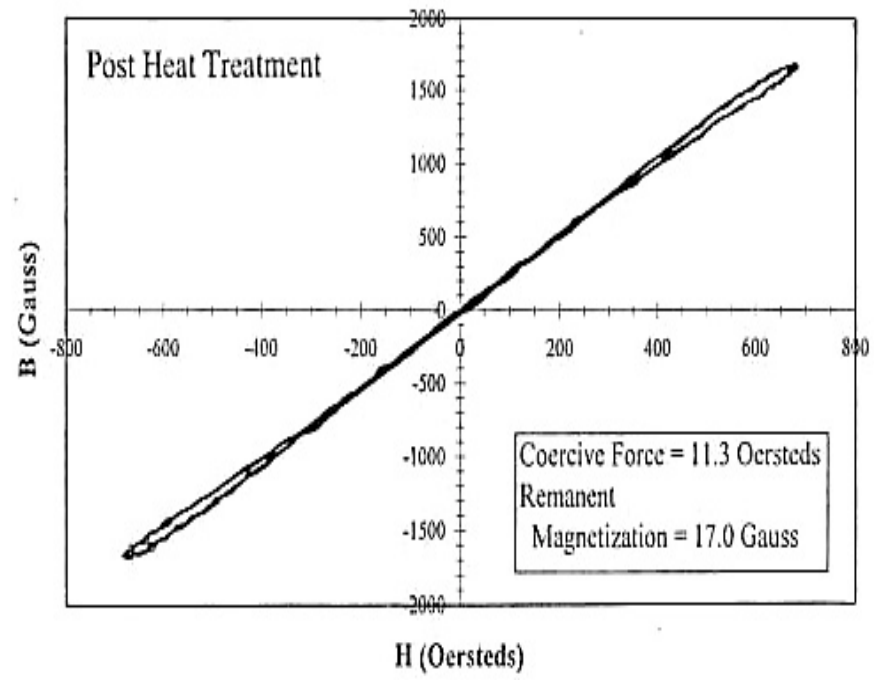

Fig. 2. A plot of the axial hysteresis loop for the transducer ring subsequent to heat treatment, showing a notable decrease in axial coercive force and retentivity. 
Torque Load Sensitivity Plot

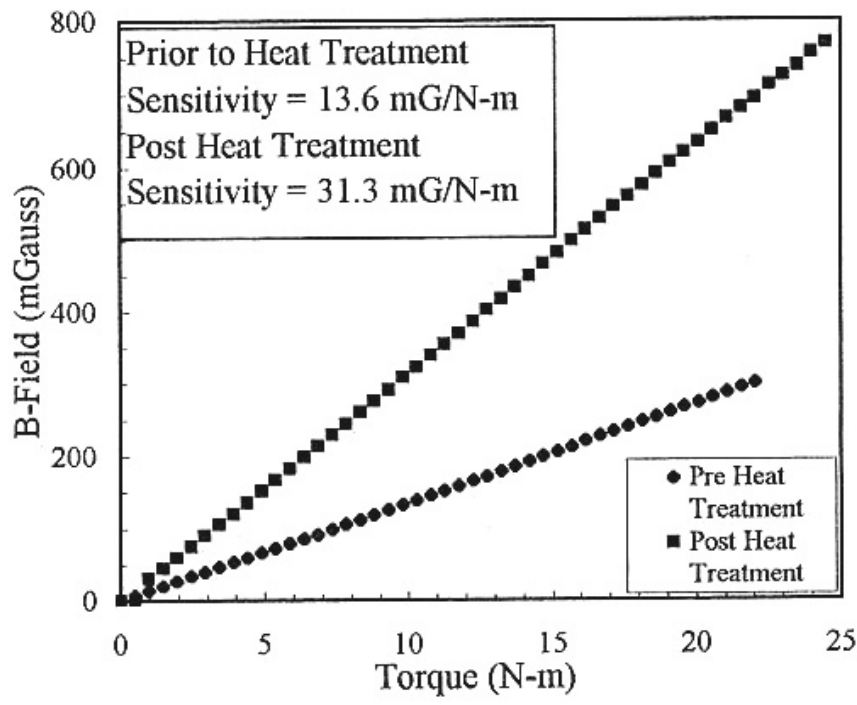

Fig. 3. Dependence of the transducer field signal response on the applied torque, exhibiting an increased sensitivity subsequent to the heat treatment.

17.0 Gauss, of the rings. This substantial decrease in the axial hysteresis parameters should directly contribute to the ability of the applied torque (shearing stress) to tilt the originally circumferential orientation of the magnetization in the transducer ring into the helical orientation as previously described, which produces the transducer's detectable axial field signal. This should be borne out by the observation of enhanced transducer sensitivity following heat treatment, as can be seen in Figure 3.

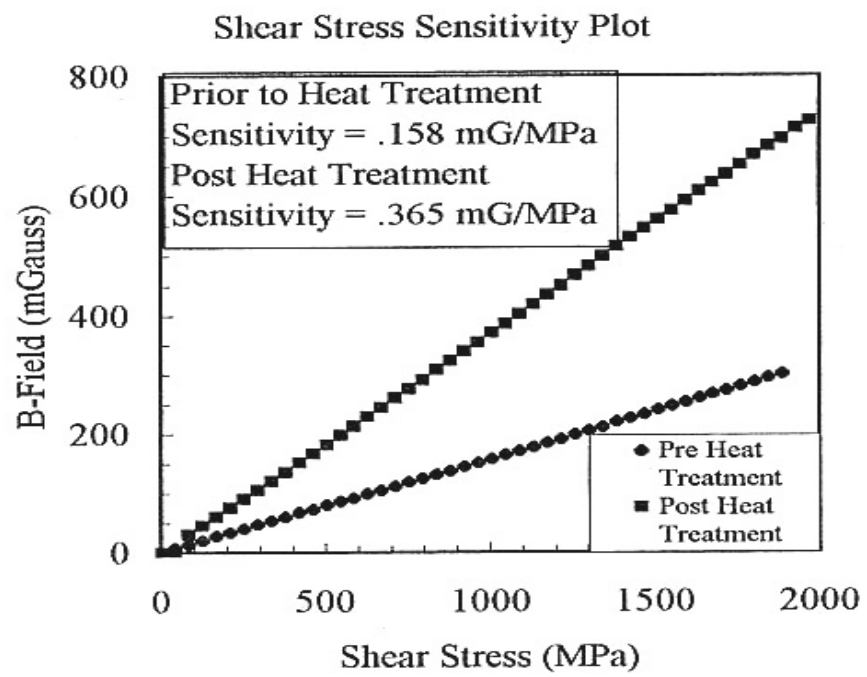

Fig. 4. Dependence of the transducer field signal response on the applied shearing stress, with these values calculated from the applied torque values.
In Figure 3, a more than twofold increase in torque transducer function, or sensitivity (field signal response to applied torque), subsequent to the heat treatments, can be noted as the rings were replaced on the shafts for torque-load testing up to applied torques of $25 \mathrm{~N}-\mathrm{m}$. The field signal response, determined by an average of linear least square fits to the data sets, dramatically increased from 13.6 to 31.3 milli-Gauss for each Newton-meter of applied torque to the ends of the shaft, and was reproducible and strikingly linear, maintaining its integrity over multiple torque cycles, and behaving consistently for increasing and decreasing torque values, in either direction. It should also be noted that within these limits of applied torque, the transducer field signal reliably returned to zero at the zero torque value, which is essential for engineering applications of the transducer. The improved sensitivity is a definite enhancement of transducer performance, resulting in improved signal to noise ratios, and minimizing the need for other forms of electronic amplification.

In order to relate the torque exerted on the shaft ends to the actual applied shearing stress within the ring itself, in Fig. 4 is plotted the field signal response in $\mathrm{mG}$ versus the shear stress (calculated from the values of applied torque) in the ring in MPa. The field signal can be seen to increase from 0.158 to $0.365 \mathrm{mG}$ for each MPa increase in applied shearing stress. This dramatic increase in the field signal for a given applied torque or shearing stress can now be directly correlated with the decrease in axial coercive force; that is, the amount of external stress needed to tilt the originally circumferential magnetic axes into a helical orientation, which results in the generation of the axial field signal, has been reduced.

In Figs. 5 and 6, respectively, are shown the circumferential hysteresis plots prior and subsequent to heat

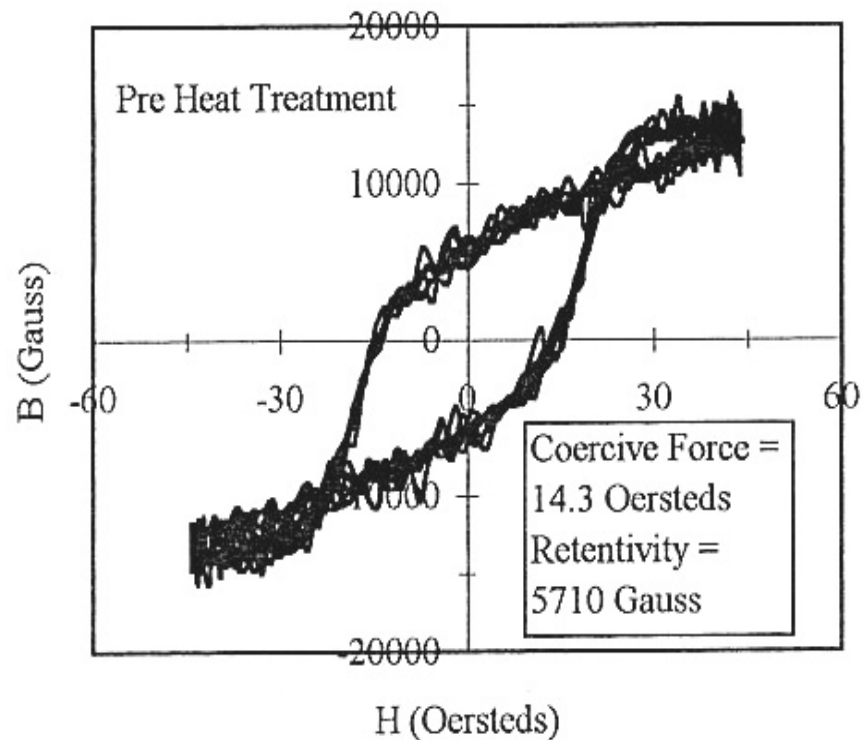

Fig. 5. A plot of the circumferential hysteresis loop for the transducer ring prior to heat treatment. 


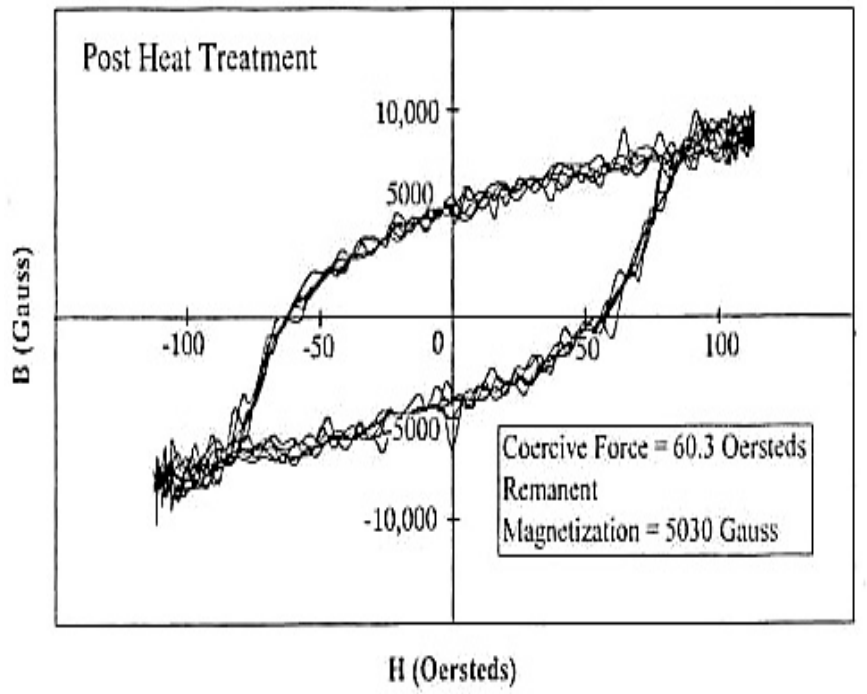

Fig. 6. A plot of the circumferential hysteresis loop for the transducer ring subsequent to heat treatment, showing a dramatic increase in circumferential coercive force.

treatment, and a significant broadening is observed in these loops. Subsequent to heat treatment, a fourfold increase in coercive force (from 14.3 to $60.3 \mathrm{Oe}$ ) was observed with little decrease in retentivity (from 5710 to 5030 Gauss). As cited in the introduction, this dramatic increase in circumferential coercive force enhances transducer stability by reducing the possible degradation of its initial circumferential magnetization by any stray fields or the axial fields which were generated by the shear stress-induced tilting of the magnetization. Thus, our overall heat treatment resulted in the successful improvement of both transducer stability and sensitivity.

The physical property changes observed to accompany the heat treatment carried out in this work can be successfully explained by a two-dimensional (axial and circumferential) model already described in an earlier publication by the authors and well-documented in the literature [5,7]. The larger sensitivity values observed subsequent to heat treatment for a given applied shearing stress correspond to larger rotations of the magnetization vector in order to achieve a minimum in the total energy per unit volume. The larger coercive force values observed after heat treatment correspond to a deeper energy minimum, and an associated enhancement of the sensor stability and integrity.

\section{Acknowledgements}

The authors acknowledge the receipt of some of the samples for this study from Magna-lastic Devices, Inc., and they also acknowledge useful technical assistance from Professor Rabchuk of the WIU Physics Department. One of the authors (MSB) acknowledges the support of the Western Illinois University Research Council. One of the authors (DKR) acknowledges the use of some physical resources from John Wood Community College, of Quincy, IL.

\section{References}

[1.] Garshelis IJ, Whitney K, May L. Development of a NonContact Torque Transducer for Electric Power Steering Systems. SAE Paper No. 920707, 1992.

[2.] Garshelis IJ, Aleksonis JA, Jones CA, Rotay RM. Development of a Magnetoelastic Torque Transducer for Automotive Transmission Applications. SAE Paper No. 970605, 1997.

[3.] Garshelis IJ, Jones CA. A Torque Transducer Based on Local Bands of Naturally Stabilized Remanent Circumferential Magnetization. J.Appl.Phys. 1999; 85: 5468-5470.

[4.] Herbst JF, Pinkerton FE. Journal of Magnetism and Magnetic Materials 1997; 176: 183-186.

[5.] Boley MS, Franklin DA, Rigsbee DK. Heat Treatment Effects on Sensitivity and Hysteresis Loops of Magnetoelastic Torque Transducers. J.Appl.Phys. 2000; 87: 7073-7075.

[6.] Bertotti G. Hysteresis in Magnetism. San Diego: Academic, 1998. p. 347.

[7.] Bozorth RM. Ferromagnetism. Princeton: Van Nostrand, 1951. p. 635-813. 